\title{
Nanofocusing parabolic refractive x-ray lenses
}

\author{
C. G. Schroer, ${ }^{\text {a) }}$ M. Kuhlmann, U. T. Hunger, T. F. Günzler, O. Kurapova, S. Feste, \\ F. Frehse, and B. Lengeler \\ II. Physikalisches Institut, Aachen University, D-52056 Aachen, Germany
}

M. Drakopoulos, A. Somogyi, A. S. Simionovici, A. Snigirev, and I. Snigireva

European Synchrotron Radiation Facility ESRF, BP 220, F-38043 Grenoble, France

C. Schug

IBM Deutschland Speichersysteme GmbH, Hechtsheimer Str. 2, D-55131 Mainz, Germany

W. H. Schröder

Institut Phytosphäre (ICGIII), Forschungszentrum Jülich, D-52425 Jülich, Germany

(Received 18 October 2002; accepted 13 January 2003)

\begin{abstract}
Parabolic refractive $\mathrm{x}$-ray lenses with short focal distance can generate intensive hard $\mathrm{x}$-ray microbeams with lateral extensions in the $100 \mathrm{~nm}$ range even at a short distance from a synchrotron radiation source. We have fabricated planar parabolic lenses made of silicon that have a focal distance in the range of a few millimeters at hard x-ray energies. In a crossed geometry, two lenses were used to generate a microbeam with a lateral size of $380 \mathrm{~nm}$ by $210 \mathrm{~nm}$ at $25 \mathrm{keV}$ in a distance of $42 \mathrm{~m}$ from the synchrotron radiation source. Using diamond as the lens material, microbeams with a lateral size down to $20 \mathrm{~nm}$ and below are conceivable in the energy range from 10 to 100 keV. () 2003 American Institute of Physics. [DOI: 10.1063/1.1556960]
\end{abstract}

Hard x-ray scanning microscopy and microprobe techniques, such as microfluorescence mapping and tomography, microdiffraction, and micro-absorption spectroscopy, rely on intensive $\mathrm{x}$-ray microbeams. ${ }^{1}$ They have greatly benefited from the high brilliance of third generation synchrotron radiation sources and recent developments in hard x-ray optics. The resolution of these techniques is limited by the lateral size of the microbeam, that typically lies in the micrometer range and that is routinely achieved at many beamlines with various hard $\mathrm{x}$-ray optics. ${ }^{2,3}$ There is, however, a great demand for x-ray analytics on the $100 \mathrm{~nm}$ scale, in particular in nanotechnology. To reach lateral beam dimensions in the 100 $\mathrm{nm}$ range is by far more demanding, but has been achieved in several recent experiments. ${ }^{4,5}$ These focusing experiments required a particularly long beamline (e.g., $145 \mathrm{~m}$ or $1 \mathrm{~km})^{5}$ or a small secondary source (e.g., $35 \times 35 \mu \mathrm{m}^{2}$ slits) in a sufficiently large distance (e.g., $43.5 \mathrm{~m}$ ) from the microbeam setup, ${ }^{4}$ since the focal length of $0.1 \mathrm{~m}$ or more does not allow one to demagnify a third generation synchrotron radiation source into the range of $100 \mathrm{~nm}$ with source to experiment distances of 30-60 $\mathrm{m}$ available at shorter beamlines.

In this letter, we present parabolic refractive $\mathrm{x}$-ray lenses with focal distances in the range of a few millimeters. With these nanofocusing lenses (NFLs) microbeams with lateral size in the range of $100 \mathrm{~nm}$ are obtainable at short distances (e.g., $42 \mathrm{~m}$ ) from relatively large synchrotron radiation sources [e.g., horizontal full width half maximum (FWHM) source size $900 \mu \mathrm{m}]$. They allow for nanofocusing at many of the shorter microprobe beamlines at third generation synchrotron radiation sources. We have successfully tested and used these lenses for microdiffraction and fluorescence tomography. Potentially, they can have diffraction limits down

${ }^{\text {a)} E l e c t r o n i c ~ m a i l: ~ s c h r o e r @ x r a y-l e n s . d e ~}$ to $20 \mathrm{~nm}$ and below in the energy range from 10 to $100 \mathrm{keV}$.

To obtain these short focal distances, lens curvatures in the micrometer range are required. To fabricate lenses with extremely small radii of curvature, the fabrication techniques for rotationally parabolic lenses previously developed in Aachen cannot be applied. ${ }^{3}$ Instead, we have used microfabrication techniques to make the planar silicon parabolic refractive $x$-ray lenses ${ }^{6}$ shown in Fig. 1. As in previous lens designs, a number $N$ of individual lenses [see shaded area (a) in Fig. 1] is aligned along a common optical axis (shown as a dashed line in Fig. 1) to form a NFL [shaded area (b) in Fig. 1]. The current design comprises lenses with $N=50$ and $N=100$. For each $N$, a set of lenses with radius of curvature varying from $R=1 \mu \mathrm{m}$ to $R=2.8 \mu \mathrm{m}$ in steps of $0.1 \mu \mathrm{m}$ is implemented on the same silicon wafer. The thickness of individual lenses on the optical axis is $d=3 \mu \mathrm{m}$. The length of the NFL is $l=8.4 \mathrm{~mm}$ for $N=100$ and $l=4.2 \mathrm{~mm}$ for $N$

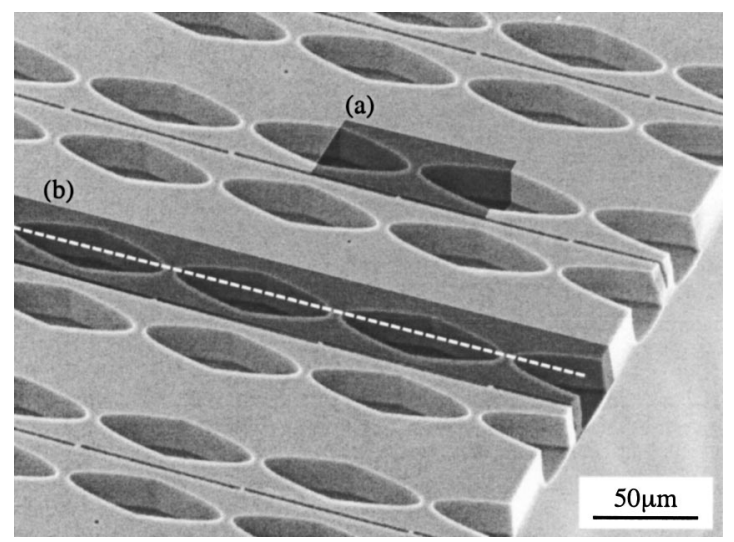

FIG. 1. Scanning electron micrograph of an array of parabolic refractive $\mathrm{x}$-ray lenses made of silicon. The shaded areas (a) and (b) delineate an individual and a compound NFL, respectively. The optical axis of the NFL is shown as a white dashed line. 
$=50$. The resulting geometric aperture $2 R_{0}$ varies from 18 to $30 \mu \mathrm{m}$. Each of these lenses produces a line focus at a slightly different distance, allowing one to realize a variety of microbeam geometries at different energies. In order to obtain two-dimensional focusing, two lenses are placed behind each other in a crossed geometry, one focusing vertically, the other one focusing horizontally. The lenses are arranged along the optical axis such that both foci coincide in one point. The common aperture is defined by a pinhole placed in front of the two lenses.

The lenses are fabricated using electron (e)-beam lithography and deep trench reactive ion etching. In a first step, the lens pattern is written into a $\mathrm{Cr}$ mask (thickness $30 \mathrm{~nm}$ ) by e-beam lithography and subsequent wet etching. The $\mathrm{Cr}$ mask is then used to transfer the pattern (using reactive ion etching) into an underlying 300-nm-thick silicon dioxide layer that serves as mask for the deep trench reactive ion etching process. With this process the lens structure is imprinted into the underlying bulk silicon. The depth of the lenses is larger than $20 \mu \mathrm{m}$.

The lenses were tested at beamline ID22 of the European Synchrotron Radiation Facility (ESRF) in Grenoble, France. The synchrotron radiation was produced by an in-vacuum undulator (third harmonic at $E=25 \mathrm{keV}$ ). The high energy content of the beam was removed using a flat Pt mirror (cutoff energy $32 \mathrm{keV}$ ). The reflected beam was monochromatized at $E=25 \mathrm{keV}$ using a cryogenically cooled fixed exit double crystal monochromator (Si 111). The lens system was setup at $L_{1}=42.1 \mathrm{~m}$ from the source. The alignment of both lenses to their common optical axis and focal plane was done using 10 degrees of freedom, observing the lenses in transmission using a high resolution x-ray camera. A PtIr pinhole (diameter $10 \mu \mathrm{m}$ ) was centered around the lenses' common optical axis to shadow radiation impinging onto the NFLs outside their aperture.

The horizontally focusing lens $(N=100)$ produced its focus at an image distance $L_{2 h}=15.6 \mathrm{~mm}$ as measured from the center of the lens. This corresponds to a radius of curvature of $R=2.15 \mu \mathrm{m}$ (design value $R=2.0 \mu \mathrm{m}$ ). The vertically focusing lens $(N=50)$ generated the vertical focus $L_{2 v}=26.7 \mathrm{~mm}$ behind its center, corresponding to a radius of curvature of $R=2.0 \mu \mathrm{m}$ (design value $R=1.7 \mu \mathrm{m}$ ). ${ }^{7}$

The lateral size of the microbeam was measured by scanning a gold knife-edge through the beam both horizontally and vertically, recording the fluorescence radiation in an energy dispersive detector. Figures 2(a) and 2(b) show the vertical and horizontal scans through the microbeam, respectively. The measured data are depicted by full diamonds. The error bars indicate the statistical error for each data point. The sum of two error functions was fitted to the data. The horizontal and vertical fits are shown in Figs. 2(a) and 2(b) as full lines. The resulting beam profiles, the derivatives of these fits, are depicted as dashed lines.

A FWHM beam size of $B_{v}=(210 \pm 50) \mathrm{nm}$ and $B_{h}$ $=(380 \pm 90) \mathrm{nm}$ are extracted from the vertical and horizontal fit, respectively. While the measured horizontal focus size is only slightly larger than the expected beam size of $340 \mathrm{~nm}$, the vertical beam size is significantly larger than the expected $110 \mathrm{~nm} .{ }^{8}$ This broadening of the focus may be explained by spherical aberrations ${ }^{9}$ due to deviations of the lens Downloaded 21 Dec 2006 to 134.94.122.39. Redistribution subject

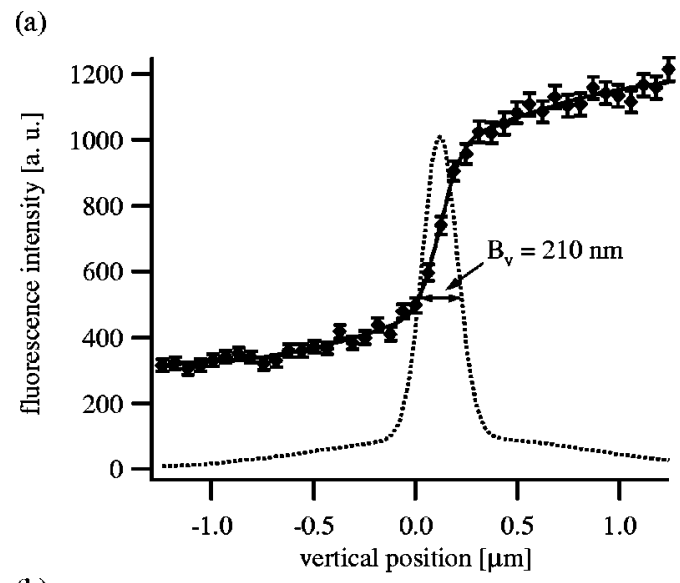

(b)

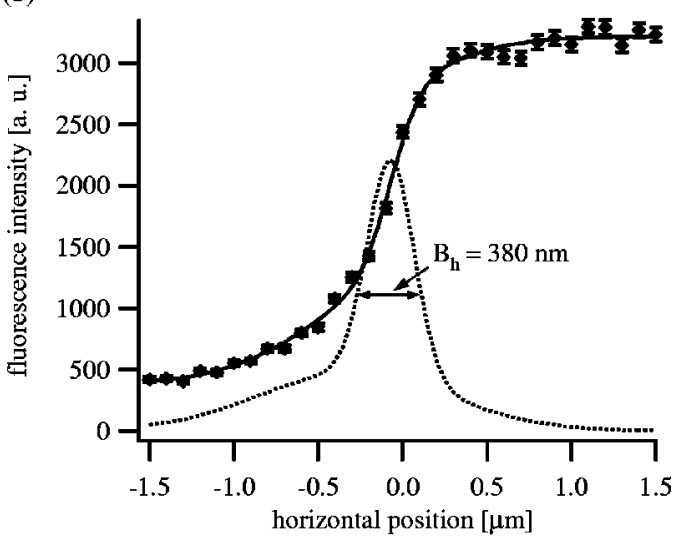

FIG. 2. (a) Vertical and (b) horizontal scan of a gold knife-edge through the microbeam.

shape from an ideal parabola. This is also suggested by the deviations of the measured radii of curvature from their design values that are most pronounced for the vertically focusing lens. These aberrations could also explain the radiation background around the focus. The asymmetry of this background in Fig. 2(b) may be due to misalignment. In future experiments improved mechanics will be required to keep the microbeam stable on the scale of the high lateral resolution.

The intensity incident on the lens was measured to $I_{0}$ $=4.3 \times 10^{12} \mathrm{ph} / \mathrm{s} / \mathrm{mm}^{2}$. Behind the lens, the integral flux was $F_{\text {total }}=4.4 \times 10^{8} \mathrm{ph} / \mathrm{s}$. From Fig. 2 the radiation inside a 1 $\times 1 \mu \mathrm{m}^{2}$ area around the focus is estimated to be $28 \%$ of the total radiation $F_{\text {total }}$ recorded behind the lens. Therefore, the flux in the focus amounts to $F_{\text {focus }}=1.2 \times 10^{8} \mathrm{ph} / \mathrm{s}$ as compared to $2.1 \times 10^{8} \mathrm{ph} / \mathrm{s}$ expected from the design parameters. The discrepancy can be explained by the halo around the focus extending beyond its ideal lateral limits. The relatively high integral flux behind the lens outside the focus is due to the relatively high transparency of the absorptive part of the PtIr pinhole (maximal thickness $80 \mu \mathrm{m}$ ) at $25 \mathrm{keV}$ that transmits about $0.1 \%$ of the incident intensity. In future experiments, more care has to be taken to define the aperture of the lens.

Besides obvious technical improvements needed to optimize the lens performance, the lens material and parameters can be chosen more systematically. To obtain minimal diffraction limits, the numerical aperture ${ }^{3} \mathrm{NA}=D_{\text {eff }} / 2 L_{2}$ needs to be maximal. Here, $D_{\text {eff }}$ is the effective aperture. ${ }^{3}$ Figure 3 shows the ideal diffraction limit $d_{t}$ (FWHM size of the Airy o AIP license or copyright, see http://apl.aip.org/apl/copyright.jsp 


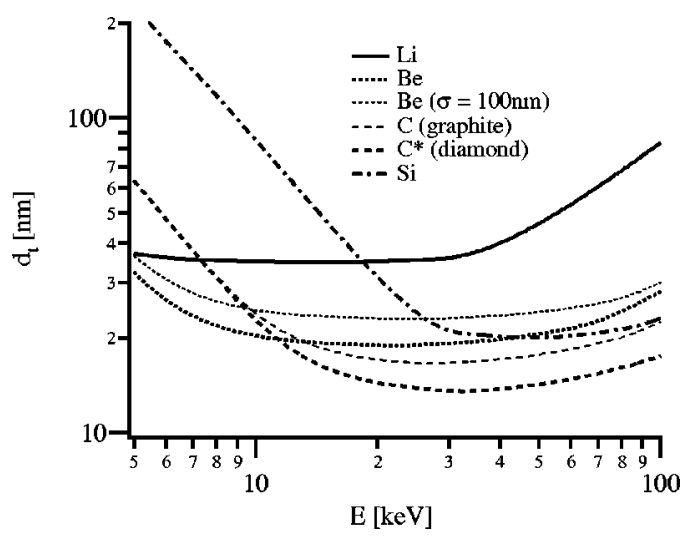

FIG. 3. Minimal diffraction limits $d_{t}$ for different lens materials as a function of x-ray energy $(N=100, l=8.4-50 \mathrm{~mm}, R=0.5-50 \mu \mathrm{m}$, other parameters were kept as in the experiment). The results were calculated using ray-tracing techniques and verified using Fresnel propagation.

disk $)^{3}$ for various lens materials, such as lithium $(\mathrm{Li})$, beryllium $(\mathrm{Be})$, graphite $(\mathrm{C})$, diamond $\left(\mathrm{C}^{*}\right)$, and silicon $(\mathrm{Si})$ as a function of x-ray energy. For this short lens design, the numerical aperture is dominated by the geometric aperture for low absorbing materials (above $10 \mathrm{keV}$ for carbon and 20 $\mathrm{keV}$ for $\mathrm{Si}$ ) and not by absorption in the outer parts of the lens, which is usually the case for refractive lenses with large focal distances. Therefore, NA is proportional to $\sqrt{\delta}$ rather than $\sqrt{\delta / \mu}$. Here, $\delta$ is the decrement of the index of refraction $(n=1-\delta)$ and $\mu$ the attenuation coefficient. Diamond that has the highest density and thus the strongest refraction of the materials shown in Fig. 3 yields the lowest diffraction limit. Lithium, however, that is often quoted as the best lens material due to its superior ratio of $\delta / \mu$, is not well suited for these lenses due to its low density and weak refraction (see Fig. 3). Relatively large surface roughness only slightly deteriorates the diffraction limit ${ }^{3}$ as shown in Fig. 3 for Be with an exaggerated rms surface roughness of $\sigma=100 \mathrm{~nm}$. For the silicon lenses an rms roughness of $\sigma<20 \mathrm{~nm}$ was experimentally achieved. The resulting broadening effects of the focus are negligible. While the surface roughness that is uncorrelated from one individual lens to the next has little impact on the imaging properties, a correlated shape error can lead to significant spherical aberration.

If the earlier experiment would be carried out with a diamond lens under ideal conditions, a nanobeam with a flux of $3.5 \times 10^{9} \mathrm{ph} / \mathrm{s}$ and a lateral size of $340 \times 47 \mathrm{~nm}^{2}$ would be expected. The gain in flux would be $8.4 \times 10^{4}$ fold. The size of the microbeam can be decreased further by reducing the length of the lens and with it the focal distance. However, there are practical limits to this: the aperture of the lens decreases and the working distance between lens and sample becomes smaller and smaller. Choosing reasonable parameters $(l=8.4 \mathrm{~mm}$, working distance $2.1 \mathrm{~mm})$, an ideal diamond lens would yield a nanobeam with $10^{9} \mathrm{ph} / \mathrm{s}$ and a lateral size of $140 \mathrm{~nm} \times 30 \mathrm{~nm}$ in the first hutch of beamline ID22 of the ESRF. At other beamlines, close to diffraction limited focusing would be possible. Diamond lenses are currently under development.

The authors thank N. Zichner for the deep trench reactive ion etching.

${ }^{1}$ G. Xu, X. Su, C. B. Stagarescu, D. E. Eastman, B. Lai, Z. Cai, I. C. Noyan, and C.-K. Hu, Appl. Phys. Lett. 78, 820 (2001); S. Bohic, A. Simionovici, A. Snigirev, R. Ortega, G. Devès, D. Heymann, and C. G. Schroer, ibid. 78, 3544 (2001); P. G. Evans, E. D. Isaacs, G. Aeppli, Z. Cai, and B. Lai, Science 295, 1042 (2002).

${ }^{2}$ U. Lienert, C. Schulze, V. Honkimäki, T. Tschentscher, S. Garbe, O. Hignette, A. Horsewell, M. Lingham, H. F. Poulsen, N. B. Thomsen, and E. Ziegler, J. Synchrotron Radiat. 5, 226 (1998); A. Iida and K. Hirano, Nucl. Instrum. Methods Phys. Res. B 114, 149 (1996); D. Bilderback, S. A. Hoffman, and D. Thiel, Science 263, 201 (1994); N. Kamijo, Y. Susuki, M. Awaji, A. Takeuchi, H. Takano, T. Ninomiya, S. Tamura, and M. Yasumoto, J. Synchrotron Radiat. 9, 182 (2002); and many articles in I. McNulty, Proc. SPIE 4499 (2001).

${ }^{3}$ B. Lengeler, C. G. Schroer, M. Richwin, J. Tümmler, M. Drakopoulos, A. Snigirev, and I. Snigireva, Appl. Phys. Lett. 74, 3924 (1999); B. Lengeler, C. Schroer, J. Tümmler, B. Benner, M. Richwin, A. Snigirev, I. Snigireva, and M. Drakopoulos, J. Synchrotron Radiat. 6, 1153 (1999).

${ }^{4}$ W. Yun, B. Lai, Z. Cai, J. Maser, D. Legnini, E. Gluskin, Z. Chen, A. A. Krasnoperova, Y. Vladimirsky, R. Cerrina, E. Di Fabrizio, and M. Gentili, Rev. Sci. Instrum. 70, 2238 (1999).

${ }^{5}$ O. Hignette, G. Rostaing, P. Cloetens, A. Rommeveaux, W. Ludwig, and A. Freund, Proc. SPIE 4499, 105 (2001); K. Yamauchi, K. Yamamura, H. Mimura, Y. Sano, A. Saito, A. Souvorov, M. Yabashi, K. Tamasaku, T. Ishikawa, and Y. Mori, J. Synchrotron Radiat. 9, 313 (2002).

${ }^{6}$ V. Aristov, M. Grigoriev, S. Kuznetsov, L. Shabel'nikov, V. Yunkin, T. Weitkamp, C. Rau, I. Snigireva, A. Snigirev, M. Hoffmann, and E. Voges, Appl. Phys. Lett. 77, 4058 (2000); V. Aristov, M. Grigoriev, S. Kuznetsov, L. Shabel'nikov, V. Yunkin, M. Hoffmann, and E. Voges, Opt. Commun. 177, 33 (2000).

${ }^{7}$ For the lenses considered here, the thin lens approximation no longer holds. All calculations in this letter include thick lens effects.

${ }^{8}$ For the calculation of the expected microbeam size, an effective source size of $900 \mu \mathrm{m}$ (horizontally) by $60 \mu \mathrm{m}$ (vertically) was assumed, given by measurements with other optics.

${ }^{9}$ The proximity effect in the e-beam lithography step and slight underetching by the deep trench reactive ion etching process introduce a shift of the lens surface, resulting in a larger radius of curvature and spherical aberrations. While we attempted to compensate for these effects, the experiment suggests that further corrections are needed to generate an ideal parabolic lens shape. 